\title{
CALIDAD DE VIDA DEL PROFESIONAL DE ENFERMERIA QUE LABORA EN LAS UNIDADES RENALES DE BOGOTA D.C.
}

Mercedes Barrera, Haydé Martinez. Sandra Rodriguez*

\section{RESUMEN}

Esta investigación describe el significado de calidad de vida y la forma como el trabajo en las unidades renales afecta a un grupo de profesionales de enfermería. Además, determinó las condiciones personales, familiares, laborales, sociales, económicas, de salud y emocionales, y aporta elementos para enriquecer futuros trabajos de calidad de vida en profesionales de enfermería. La metodología utilizada fue cuali-cuantitativa con un diseño descriptivo transversal. La muestra fue de 28 profesionales de enfermería de 14 unidades renales de Bogotá que accedieron voluntariamente a participar en la investigación, luego de que los coordinadores aceptaron la realización del estudio.

Como antecedente y referencia del trabajo, se tuvo en cuenta el estudio de calidad de vida en médicos y odontólogos generales en Santafé de Bogotá 1998-1999.

I ,os resultados mostraron una población de sexo femenino en su mayoría, jóvenes, casadas, con uno a dos hijos, en modalidad de contrato con entidades privadas y con vinculación de planta en jornadas laborales de ocho horas. Su remuneración varió entre uno y dos millones de pesos, invertidos en educación, cuota de vehículo, arrendamiento, alimentación y servicios públicos, entre otros. Generalmente asisten a consulta médica inducidos por la enfermedad. Las causas de morbilidad fueron, en su orden, las afecciones respiratorias, la enfermedad ácido péptica, las enfermedades posturales y la depresión. La automedicación está presente en la mayoría de los encuestados, en especial para buscar analgesia. EI sueño fue placentero pero no recuperador, y el consumo de tinto como hábito no protector mostró un aumento significativo. En cuanto a aspectos sicológicos y emocionales, los encuestados tienen una percepción positiva de su imagen con presencia de sentimientos positivos, aunque consideran que hay muy poco apoyo estatal y empresarial para el aprovechamiento de dicho profesional.

Con respecto a la pregunta sobre el significado de calidad de vida, para un poco más de la mitad de los encuestados incluyó la palabra bienestar, a nivel físico, económico, emocional, social, laboral y de salud. La forma como el trabajo influye en la calidad de vida incluyó respuestas positivas y negativas como poco tiempo libre, incapacidad de compartir con la familia, jornadas de trabajo largas y rutinarias, y agotamiento. También consideraron que es un buen campo de trabajo, se aprende, es motivante, genera un salario y es productivo.

Palabras claves: calidad de vida, enfermeras y unidades de diálisis renal en hospital.

\section{Introducción}

El acto del cuidado en enfermería tiene un carácter especialmente humano. Coordina y hace efectivo el conocimiento científico y tecnológico en áreas tan especiales como las unidades renales, lugares de trabajo son especialmente complejos dados la enfermedad de los pacientes, las exigencias y el estrés al que diariamente

Egresadas del programa de especialización en Nefrologia y Urologia. Fundación Universitaria de Ciencias de la Salud. Hospital de San José. se ven afectados, donde el imperativo ético de cuidar del otro, que deriva de la profesión, exige también el cuidar de sí mismo.

La calidad de vida en los profesionales de enfermería de las unidades renales fue de interés para las autoras, pues se considera que es una aspiración y un derecho y es un elemento esencial para desarrollar con eficacia la función asignada a este colectivo dentro de la comunidad, que exige mayores y mejores prestaciones de servicio y de cuidado. El profesional ejerce una 
influencia directa sobre las personas a las que cuida. Por lo tanto, su propia calidad de vida en todos los aspectos (comodidad laboral, condiciones de trabajo, estado de ánimo, hábitos protectores, entre otros), repercute también en las personas a las que atiende durante el día.

Es importante identificar, analizar y buscar, a nivel individual y grupal, el establecimiento y el mantenimiento de buenas condiciones personales, familiares y de trabajo que sean económica y socialmente justas, y que promuevan su desarrollo integral y favorezcan en gran parte su calidad de vida.

\section{El profesional de enfermería en las unidades renales}

La interacción entre el profesional de enfermería y el paciente de la unidad renal está mediada permanentemente, tanto por la necesidad de cuidado directo relacionado con el tratamiento específico como por las necesidades de información y educación del enfermo y su familia, que abarcan desde los problemas amplios y complejos hasta asuntos de la cotidianidad derivados de su situación de enfermedad.

La hemodiálisis y la diálisis peritoneal crean un vínculo especial entre el profesional de enfermería y el paciente, basado en la confianza personal y profesional. El profesional no sólo cuida del paciente sino que también se convierte en su continuo soporte maestro y en motivador de la rehabilitación. A medida que pasa el tiempo, el estado del paciente se estabiliza, el proceso de asistencia incita al paciente a la participación en los cuidados, y se desarrolla una independencia y un sentimiento de mayor autodominio.

El continuo intercambio de ideas, e incluso de sentimientos, es fundamental para conseguir que pacientes que en un principio estaban condenados a morir, vivan y lo hagan con un grado de rehabilitación adecuado, que les permita no considerarse ni siquiera enfermos.

El profesional de enfermería se comunica con el paciente antes de llegar a la situación de fracaso crónico terminal, y desempeña un papel importante para que asuma su situación con realismo y elija entre las diver- sas opciones de tratamiento (hemodiálisis, diálisis peritoneal o trasplante renal).

La diálisis remueve toxinas de desecho y el exceso de líquidos del cuerpo; pero otras funciones como el control de la tensión arterial, el metabolismo de la vitamina $\mathrm{D}$ y la producción de glóbulos rojos deben ser alcanzadas con terapias farmacológicas. Las restricciones en la dieta y en los líquidos, junto con los problemas sociales y psicosociales, son comunes a lo largo del tratamiento. Por lo tanto, el profesional de enfermería en la unidad renal requiere un rango de atributos entre los que se encuentran habilidad técnica, conocimientos científicos, destrezas de enseñanza y capacidad de proporcionar soporte emocional.

En las unidades renales las enfermeras deben desarrollar otras habilidades como el liderazgo, la toma de decisiones, el manejo adecuado del estrés derivado de su trabajo, y la búsqueda del equilibrio y la armonía en su vidaque les permitan tener una buena calidad de vida.

\section{Metodología}

Es un estudio descriptivo transversal de diseño cualicuantitativo, que tomó como base el profesional de enfermería que labora en 14 unidades renales de Bogotá. La muestra fue de 28 profesionales. Con autorización previa de las instituciones y asegurando la confiabilidad y confidencialidad, el grupo investigador decidió, como criterio de inclusión, una experiencia mínima de seis meses.

Las unidades renales escogidas fueron la Fundación Hospital San Carlos, la Clínica San Rafael, el Hospital Universitario de la Samaritana, el Hospital Militar Central, el Hospital de San José, la Clínica Nueva, la Clínica San Pedro Claver, el Hospital San Ignacio, el Hospital Central de la Policía, el Instituto Nacional del Riñón, la Fundación Santa fé de Bogota, la Fundación Cardio Infantil, la Cruz Roja Colombiana y Nuevo Horizonte.

Es de interés para el lector saber qué métodos se aplicaron las autoras para la entrevista, qué preguntaron, como lo hicieron, qué instrumento utilizaron, etc. 


\section{Resultados}

La muestra estuvo conformada por 27 mujeres y 1 hombre, en su mayoría menores de 40 años, procedentes de Bogotá, con vínculo matrimonial y con uno o dos hijos.

Laboralmente se encontró un grupo de profesionales vinculados de planta, en su mayoría, y otros, en menor número, por contrato principalmente por entidades privadas. Esta área de trabajo posee estabilidad laboral y variedad de horarios, lo que permite el multiempleo, ejercido por un pequeño grupo de los encuestados y que evidencia la necesidad de aumentar el poder adquisitivo por parte del profesional.

La mayoría de las enfermeras mancionó el poder y el liderazgo como algo que ejercen siempre o casi siempre. Este hallazgo es interesante, dado que el trabajo en áreas tan especiales como las unidades renales requiere lideres para la organización, control de actividades, y amerita el trabajo de personas con capacidad para tomar decisiones.

En cuanto al reconocimiento a la labor, la mitad de los encuestados respondió queexistía siempre o casi siempre.

La remuneración proveniente del trabajo es determinante para la satisfacción de las necesidades básicas y la motivación del desempeño laboral. Este se distribuye mensualmente, en su orden de frecuencia, para educación, cuota de vehículo, arrendamiento, alimentación, cuota de vivienda, serviciospúblicos, vestuario, recreación, salud y pago de tarjetas de crédito.

Con relación a los seguros de salud, el total de los encuestados está afiliado a una EPS, y algunos tienen cobertura adicional con medicina prepagada y seguro de vida. En cuanto a la percepción de salud, la mayoría se siente bien, y menos de la mitad de los encuestados asistio a consulta médica en los últimos seis meses inducidos por enfermedad o de forma voluntaria.

Las causas de morbilidad fueron, en su orden, las enfermedades respiratorias, la enfermedad ácidopéptica, las alteraciones posturales, la depresión y las dislipidemias. Pudo apreciarse su relación con factores ocupacionales.
Respecto al consumo de medicamentos, se apreció que la auto medicación está presente en la mayoría de los encuestados, especialmente de analgésicos.

Respecto al sueño, se observó que fue placentero en su mayoría y recuperador en la minoría, lo cual demuestra que un alto porcentaje de los encuestados no logra un verdadero descanso.

En cuanto al número de horas de descanso, se observa que en la mayoría de los encuestados predominó el rango de 5 a 7 horas.

La mayoría de profesionales tuvo poco consumode alcohol y cigarrillo. El cafémostró un aumento en menos de la mitad, lo que se podría relacionar como uso de una estrategia negativa.

El tiempo libre es de gran importancia para el total de los encuestados, y está dedicado a actividades como lectura, cine, recogimiento, recreación, deporte, viajes, y actividades culturales.

En cuanto a aspectos psicológicos y emocionales, los profesionales encuestados tuvieron una percepción positiva de su imagen. En la mayoría hubo sentimientos positivos. Una minoría expresó sentimientos negativos.

Desde el año 1995 se ofrece la especialización de Enfermería en Nefrología y Urología en la Fundación Universitaria de Ciencias de la Salud, de la cual han egresado 32 profesionales. Del grupo de encuestados, una minoría tiene esta especialización, dato interesante si se analiza cuál es el área de desempeño de los profesionales especializados.

El significado de calidad de vida, para un poco más de la mitad de los encuestados, incluyó la palabra bienestar a nivel físico, económico, emocional, social, laboral y de salud.

En relación a cómo interfiere el trabajoen la calidad de vida, hubo respuestas positivas y negativas. Influyó de manera negativa porque interfiere con el tiempo libre, impide compartir con la familia, incluye largas jornadas de trabajo, y es rutinario y agotador. Menos de la mitad de los encuestados respondió que presenta estrés por exceso de trabajo, situaciones complicadas de los pacientes, la responsabilidad y el alto riesgo de complicaciones durante el cuidado de los usuarios. 
Dentro de las respuestas positivas, los encuestados manifestaron que el trabajo actúa como un aprendizaje, una experiencia laboral en la búsqueda de satisfacción personal y genera productividad. Es una forma de motivación que genera un salario para cubrir necesidades básicas, y es un buen campo de trabajo.

\section{Conclusiones y recomendaciones}

La enfermera, dentro y fuera de su trabajo, debe velar para que la salud y la calidad de vida mejoren, efectuando propuestas coherentes y viables. Debe procurar la utilización de medidas preventivas y estilos de vida saludables que lleven al mantenimiento de un buen estado de salud tanto físico como emocional.

Debe revisar los sistemas de trabajo diario y buscar soluciones para eliminar procesos rutinarios, sistematizar y optimizar el manejo de registros, dando prioridad aquellos propios de la profesión, para utilizar el tiempo y la energía al cuidado del usuario.

Debe trabajar continuamente en el mejoramiento de su calidad de vida, buscando el bienestar, el equilibrio y la armonía.

Los profesionales reciben poco apoyo estatal, por lo que se recomienda impulsar la creación de una sociedad científica de enfermeras en Nefrología y Urología, que lidere a nivel nacional y regional el mejoramiento de la calidad en el cuidado, la protocolización y la evaluación permanente, y que propenda por mejorar las condiciones laborales y los estímulos.

\section{Se recomienda:}

Permitir la asistencia del personal a cursos de actualización en los avances científicos-técnicos, así como a actividades de crecimiento y mejoramiento personal como las recreativas, o en técnicas de relajación.

Fomentar la participación de las enfermeras en los procesos de cambio y mejoramiento de las unidades renales, así como estimular la comunicación y el liderazgo.
Desarrollar programas de educación continua acordes a las necesidades del personal, que propendan por el mejoramiento de la seguridad y la confianza.

Ofrecer estímulos laborales ajustados a las exigencias.

\section{Sugerencias para estudios posteriores}

- Componente ocupacional y situacional de las causales de morbilidad de las enfermeras dentro de las unidades renales.

- Estrés del profesional de enfermería dentro de las unidades renales.

- Elaborar un manual de funciones teniendo en cuenta el perfil del profesional de enfermería en esta área, con el fin de unificar conceptos de cuidado directo y elabora una guía de manejo en las unidades renales de Bogotá.

\section{Bibliografía}

Abstract internet. Congreso de Calidad De Vida. Puerto Rico. 2000

Calidad de vida en médicos y odontólogos en santa fe de Bogotá D.C. 1998-1999. Tesis de Grado.

Código y tribunal de ética en enfermería

Constitución política de Colombia 1991

Ley 226 de 1996 Artículo 18. Capitulo 6

Contreras M, Hernán. Un modelo cuantitativo de calidad de vida. Nuestra Sociedad, (75) Caracas Venezuela. Enero-Febrero 1985.

Creus Virgili, Mariana. Enfermería profesión. Calidad de vida en Enfermería (condición indispensable para prestar cuidados de calidad) Revista Rol de Enfermería No154.

Franco Peláez, Zoila Desarrollo Humano y de valores en salud, Bogotá, Junio 1999. Pag 319-400.

Galache, Belén A y otros. Administración. Personal de Enfermería en las Unidades de Diálisis. Revista Rol de Enfermería No 102.

Godue, Charles. La Salud en los procesos de Globalización y de internacionalización Pag 15-50.

Grijalba de Rodado Elizabeth. Hacia una Mejor Calidad de Vida. Trabajo de grado Sicología Universidad Javeriana 1998.

Lopez Rodrigo, Martha. Enfermería Profesión. Estrés de los profesionales de Enfermería. Mayo de 1995.

Galache, Belén. Personal de Enfermería en las unidades de Diálisis Revista Rol de Enfermería N 154

Insuficiencia Renal, Diálisis y Transplantes. Grupo de Trasplantes Universidad de Antioquia, Salvat pag 25-40.

contreras M, Hernán Calidad de vida en una nueva sociedad ( I-II 1985 Venezuela) 